\title{
Gastrointestinal stromal tumor mimicking such as incarcerated inguinal
}

\section{hernia}

\author{
Murat Ferhat Ferhatoglu $^{1}$, Abdulcabbar Kartal ${ }^{1 *}$, Taner Kivilcim $^{1}$
}

\begin{abstract}
Gastrointestinal stromal tumors, are most common sarcoma of the gastrointestinal tract, originate from Cajal cells which are responsible for the motility of the gastrointestinal tract. This sarcoma is most commonly observed in stomach and small intestines, can rarely be located in omentum, mesentery or retroperitoneum. Herein, we aimed to present a case of gastrointestinal stromal tumor presented as incarcerated inguinal hernia.
\end{abstract}

Key words: Gastrointestinal stromal tumor, GIST, hernia, inguinal hernia, incarceration

\section{Introduction}

Gastrointestinal stromal tumor (GIST) is a mesenchymal tumor originating from Cajal cells responsible for the motility of the gastrointestinal tract (1). GISTs are most commonly located in stomach $(60-70 \%)$, small intestines $(20-30 \%)$, colon and rectum $(5 \%)(2)$, but it may be localized anywhere from esophagus to anus. Rarely, they can localize in retroperitoneum, omentum and mesentery(3). Primary treatment is surgical removal of the mass.

The most common structures found in the inguinal hernia are omentum and small intestines. More rarely, however, the appendix, sigmoid colon, bladder can be found in the inguinal hernia sac. It is an extremely rare case that an gastrointestinal system originated tumoral mass to be found in the inguinal hernia sac. We aimed to present a case of GIST presenting in the form of left incarcerated inguinal hernia in this case.

Case

A 67 year-old male patient was admitted to the emergency department with left groin pain and mass swelling in the left groin. An irreducible, hard, painful mass was palpated in the left groin area. He had subfebrile fever and in the blood tests leukocyte count was $11400 / \mathrm{mm} 3(4000-10400 / \mathrm{mm} 3)$, all other test result were normal. He was operated with the diagnosis of left incarcerated inguinal hernia. A necrotic mass filling up the hernia sac with a size of $13 \times 4 \mathrm{~cm}$ with hemorrhage on the surface was observed during surgery. (Figure 1) The mass was isolated from the spermatic cord and the testis and excised.
When intraabdominal site was palpated from the hernia incision during surgery, it was determined that there were widespread implants on the peritoneal surfaces. As a result of the pathologic examination, the patient was reported having GIST mass, postoperative radiological examination revealed extensive GIST metastases in the abdomen and on the peritoneal surfaces and imatinib mesylate treatment was initiated for the patient by a medical oncologist.

\section{Discussion}

GISTs are the most common sarcomas of the gastrointestinal tract. Rarely, they may be located in the abdominal peritoneum or mesentery. However GISTs located in these localizations are often presented as metastasis of the primary disease originating from the gastrointestinal tract (5).

Since they usually give symptoms in the late period, more than $50 \%$ of patients have locally advanced or metastatic disease at the time of admission (2).

Surgical removal of the mass as a whole constitutes is the basis for treatment. However, even after a complete surgical resection, $50-65 \%$ of patients have a 5 year survival chance. In advanced stage and metastatic disease, this rate is reduced to $35 \%(2)$.

Although there are publications in the literature about positivity of lymph node metastasis $(6,7)$, nodal metastasis is very rare in GIST, and thus surgical consensus is that a lymph node dissection is not necessary (8).

Received 19-03-2018 Accepted 29-03-2018 Available Online 31-03-2018

1 Okan University, Faculty of Medicine, Dept. of General Surgery, Istanbul, TR

* Corresponding Author: Murat Ferhat Ferhatoglu E-mail: ferhatferhatoglu@ gmail.com Phone: +90 (555) 3214793 


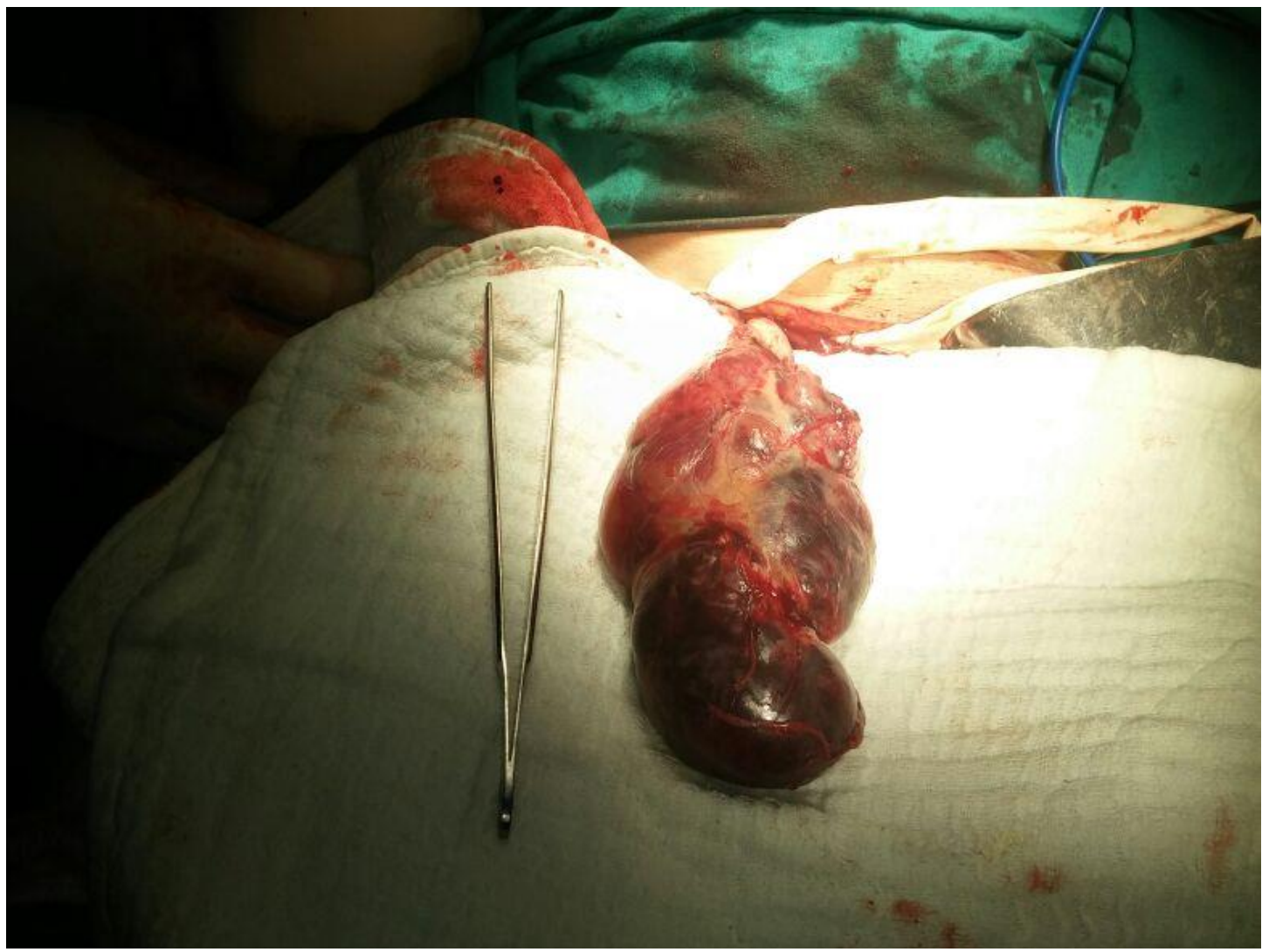

Figure 1: Hemorrhagic fragile mass taken out from the hernia sac

Table 1. Assessment of clinical aggression of GIST in accordance with the American National Institutes of Health guidelines (10)

\begin{tabular}{lcc} 
Aggressiveness & Tumor size $(\mathrm{cm})$ & Mitotic index $(\mathrm{HPF})$ \\
Very low & $<2$ & $<5 / 50$ \\
Low & $2-5$ & $<5 / 50$ \\
Moderate & $\leq 5$ & $6-10 / 50$ \\
& $5-10$ & $<5 / 50$ \\
High & $>5$ & $>5 / 50$ \\
& $>10$ & $>10 / 50$ \\
\hline
\end{tabular}

Table 2. Poor prognostic factors for GIST (1)

\begin{tabular}{ll}
\hline$\checkmark$ & Tumor size $>10 \mathrm{~cm}$ \\
$\checkmark$ & High mitotic index $(\geq 5 / 50 \mathrm{HPF})$ \\
$\checkmark$ & Non-gastric localization \\
$\checkmark$ & Distant metastasis \\
$\checkmark$ & Damage to the integrity of the tumor during surgery \\
$\checkmark$ & Perforation \\
$\checkmark$ & Multifocal tumor \\
$\checkmark$ & Advanced age \\
$\checkmark$ & Widespread tumors \\
\hline
\end{tabular}


In $95 \%$ of the cases c-kit (CD117) (4), in $70 \%$ CD34 (1), in $5 \% \square$ - platelet derived growth factor gene mutations are observed (9). In post-operative adjuvant treatment imatinib mesylate, a specific c-kit inhibitör is used. It has been shown that this treatment reduces or stops progression of the tumor in more than $50 \%$ of patients (10).

In patients with high-risk tumors (Table 1) and poor prognostic factors (Table 2), the recurrence can be reduced by routine follow-up after surgery and adjuvant chemotherapy.

A sudden, irreducible, painful mass in the inguinal region is called an incarcerated inguinal hernia. This situation is one of the few classical emergency surgical indications. The absence of inguinal hernia history, description of non-specific gastrointestinal symptoms and history of GIST surgery should make the surgeon suspect that it may be an additional pathology, and additional radiological examinations such as ultrasonography can be used to diagnose unusual pathologies preoperatively. In our case, the surgeon considered the patient as having an incarcerated inguinal hernia and during surgery, noticed that the palpated mass within the hernia sac was a tumoral mass.

\section{Conclusion}

Incidental tumor finding in the inguinal hernia sac is an extremely rare condition. Very few cases have been reported in the literature regarding this situation. Advanced technological imaging methods such as ultrasonography should be used to detect cases such as this and other rare cases in patients with a long history of gastrointestinal symptoms, especially those with no inguinal hernia history

\section{Acknowledgments, Funding: None}

Conflict of Interest: The authors declare no potential conflicts of interest with respect to the research, authorship, and/or publication of this article.

Author's Contributions: MFF, AK, TK: Research concept and design; Surgery, analysis and interpretation of data. MFF: Article preparation and revisions. All authors approved the final version of the manuscript,
Ethical issues: All Authors declare that Originality of research/article etc... and ethical approval of research, and responsibilities of research against local ethics commission are under the Authors responsibilities. The study was conducted due to defined rules by the Local Ethics Commission guidelines and audits.

\section{References}

1. Cybulka B, Golanski M, Rapela J, et al. Elective surgery of umbilical hernia as a first clinical manifestation of a gastrointestinal stromal tumor(GIST)- case report. Polski Prezeglad chirurgiczny 2016; 4: 221-25.

2. Liu PH, Kung WC, Wu YC, et al. Metastatic malignant gastrointestinal stromal tumor mimicking a right incarcerated inguinal hernia. Formosan Journal of Surgery 2014; 47: 18992.

3. Nilsson $B$, Bümming $P$, Meis- kindblom JM, et al. Gastrointestinal stromal tumors: the incidence, prevalence, clinical course, and prognostication in the preimatinib mesylate era- a population based study in sweden. Cancer. 2005; 103: 821-9.

4. Gonzalez JT. Gastrointestinal stromal tumor(GIST) presenting as a groin mass mimicking and incarcerated hernia. Int j surg case reports 2015; 6: 166-8.

5. Mulla MG, Yeung J, Reynolds JR et al. Gastrointestinal stromal tumour(GIST) mimicking an inderct inguinal hernia, a rare presentation. Eur Surg. 2007; 39: 270-2.

6. Shafizad A, Mohammadianpanah $\mathrm{M}$, Nasrolahi $\mathrm{H}$ et al. Lymph node metastasis in gastrointestinal stromal tumor(GIST): to report a case. Iran J Cancer Prev. 2014; 3: 171-4.

7. Prakash S, Sarran L, Socci N, et al. Gastrointestinal stromal tumors in children and young adults: a clinicopathologic, molecular and genomic study of 15 cases and review of the literature. J Pediatr Hematol Oncol. 2005; 27: 179-87.

8. Pierie JP, Choudry U, Muzikansky A, et al. The effect of surgery and grade on outcome of gastrointestinal stromal tumors. Arch Surg. 2001; 136: 383-9.

9. Joensuu H. Gastointestinal stromal tumor (GIST). Ann Oncol 2006; 17: 280-6.

10. Fletcher CD, Berman JJ, Corless, et al. Diagnosis of gastrointestinal stromal tumors: a consensus approach. Hum Pathol. 2002; 33: 459-65.. 